\title{
The Stability of Boolean Rules Memory Based on the Core Systems of Number-Processing
}

\author{
Xiuzhen Wang ${ }^{\mathrm{a}}$, Weiquan $\mathrm{Gu}^{\mathrm{a},{ }^{*}}$ \\ ${ }^{a}$ College of Arts and Sciences, Harbin Normal University, Harbin City, 150301, China
}

\begin{abstract}
Activation of how and where arithmetic operations are displayed in the brain has been observed in various number-processing tasks. However, it remains poorly understood whether stabilized memory of Boolean rules are associated with background knowledge. The present study reviewed behavioral and imaging evidence demonstrating that Boolean problem-solving abilities depend on the core systems of number-processing. The core systems account for a mathematical cultural background, and serve as the foundation for sophisticated mathematical knowledge. The Ebbinghaus paradigm was used to investigate learning-induced changes by functional magnetic resonance imaging (fMRI) in a retrieval task of Boolean rules.
\end{abstract}

Index Terms: stability; core system; Boolean rule; fMRI; stabilized memory

(C) 2011 Published by MECS Publisher. Selection and/or peer review under responsibility of the Research Association of Modern Education and Computer Science.

\section{Introduction}

Memory stability, the ability to retain the past information and to influence future results [1], is thought to be due to the persistence of memory information, but remains flexible in face of environmental perturbations [2]. In an educated human, experiential knowledge could provide the foundation for an integration with stability-based processing of numbers. Much of advanced mathematics is built on basic number-processing skills, which is thought to be supported by a distributed network of various brain regions, including frontoparietal (for a review, e.g., [3]) and other areas associated with various tasks [4]. A wealth of neuroimaging data $[5,6]$ has revealed that activity in the fronto-parietal areas correlates with task rules in number-processing, which was measured either by retrieving a pure arithmetic fact or by calculating a memorized multi-fact task [7]. Therefore, core systems may serve to process concepts or facts, independently of non-numerical or numerical symbols. Nevertheless, the basic ability to calculate numerical tasks cannot fully explain the extent of human mathematical skills. During learning of advanced mathematics, a large set of unique human skills

* Corresponding author.

E-mail address: guweiq.gu@gmail.com 
emerge, which provide a person with the ability to understand abstract mathematical rules (such as Boolean rules and integral calculus) and perform mathematical operations based on core systems of number.

In the present study, according to Ebbinghaus' memory paradigm [8] and the theory of working memory capacity[9], the effects of memory stability of Boolean rules based on background knowledge, as well as the relationship between Boolean rules and core systems of number-processing, were investigated. Specifically, the possibility of estimating the same regions of brain activation patterns for the mathematical thinking core was analyzed. For each problem of the seven kinds of rules in Table 1, a set of problems, such as $\mathrm{P} \uparrow \mathrm{Q} \rightarrow \mathrm{P}$ were presented during a 1-week training period. Training success was measured in decreasing reaction times and error rates. The first two kinds of rules in Table 1 served as background knowledge. In a subsequent fMRI session (day 7), trial retrieval consisted of the last five kinds of rules (every kind of rule contains 4 expressions) in Table 1. Two hypotheses are possible. First, it was assumed that the time-dependent learning utility had crucial influence, and simultaneously, that extensive and repetitive training for the rules in Table 1 should lead to similar modifications of brain activation. Second, it was assumed that characteristics inherent to the last five kinds of rules in Table 1 determine the acquisition process extensive and repetitive training should be based on number-processing and lead to an identical core of activation patterns. Based on previous studies [10], these predictions could be applied to Boolean rules. Trained rules exhibited identical activation in the left parietal and left inferior frontal regions, and serve to process the simple arithmetic operations (arithmetical facts), while the trained rules exhibited a significant focus of activation around the fronto-parietal region [11]. These experimental effects have not been previously shown. The present study summarized the experimental analysis of behavior analysis and then reported the fMRI results for the stability of Boolean rules association with the core systems of number-processing.

Table 1 Boolean rules

\begin{tabular}{|c|c|c|c|c|c|c|c|c|}
\hline $\begin{array}{l}\mathrm{C} \\
\mathrm{N}\end{array}$ & $\mathrm{R}^{\mathrm{C}}$ & $\mathrm{OR}^{\mathrm{AN}}$ & & $\overline{\mathrm{OR}}$ & IMP & $\overline{\mathrm{EQ}}$ & & NAND \\
\hline $\mathrm{P}$ & Q & $\mathrm{P} \wedge \mathrm{Q}$ & $\mathrm{P} \vee \mathrm{Q}$ & $\mathrm{P} \rightarrow \mathrm{Q}$ & $\mathrm{P} \longleftrightarrow \mathrm{Q}$ & $\mathrm{P} \uparrow \mathrm{Q}$ & $\mathrm{P} \downarrow \mathrm{Q}$ & $\mathrm{P} \nabla \mathrm{Q}$ \\
\hline 0 & 0 & 0 & 0 & 1 & 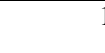 & & 1 & 1 \\
\hline $\begin{array}{l}0 \\
1\end{array}$ & 1 & 0 & 1 & 1 & ( & & 1 & 0 \\
\hline $\begin{array}{l}1 \\
1\end{array}$ & 0 & 0 & 1 & 0 & ( & ) & 1 & 0 \\
\hline $\begin{array}{l}1 \\
0\end{array}$ & 1 & 1 & 1 & 1 & 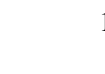 & & 0 & 0 \\
\hline
\end{tabular}

\section{Experimental Procedures}

\subsection{Participants}

Eighteen university students (9 females; mean age $23.2 \pm 2.5$ years) participated in the study. All participants were informed about the procedure and gave written consent to participate in the experiment. The study was approved by the Ethical Commission of the Xuanwu Hospital of Capital Medical University. Six subjects had to be discarded from the analysis due to technical problems with the unsuccessful data collection $(n=5)$ or excessive motion of the head $(n=1)$. Thus, fMRI image analysis is based on the data from 12 participants. 


\subsection{Training procedure}

To examine the stable processes underlying learning-dependent memory, Boolean arithmetic tasks were utilized, with two operator symbols that served as trained tasks (eg. $\mathrm{P} \uparrow \mathrm{Q} \rightarrow \mathrm{P}$ ). fMRI test tasks were similar to those of adults' learning artificial Boolean rules [12]. Subjects were trained on Boolean tasks until they were perfectly mastered. The subjects were tested by fMRI on the day before day 1, day 1 and day 7. On the practice days, participants took part in seven training sessions on consecutive days and were trained on twenty Boolean arithmetic problems. Each problem comprised of two operator symbols in a set of twenty problems. The problem remained visible until the subject finished typing the solution. When the problems were presented on a computer, subjects entered the answer ( 1 or 0$)$ using the number key on the computer keyboard. Reaction times were found starting with the presentation of the problem until the answer was provided. Incorrect (correct) response times and average percent correct feedback were provided. If accuracy was $<80 \%$, the subjects were tested on a new task set. Within a series of behavior experimental conditions, the overtime criterion was $14 \mathrm{~s}$.

Table 2 Mean response accuracies and latencies for rule retrieval on the repeat-three-times task

\begin{tabular}{|c|c|c|}
\hline & Response accuracy $(\%)$ & Reaction time (s) \\
\hline Pre-test & $45.00(1.7)$ & $2.39(0.05)$ \\
\hline Post-test & $95.97(0.7)$ & $2.35(0.04)$ \\
\hline Long-test & $97.78(0.5)$ & $1.66(0.04)$ \\
\hline
\end{tabular}

Table 3 Regions of activation (in talairach space) during all the retrieval rules relative to rest (intertrial interval)

\begin{tabular}{|c|c|c|ccc|c|c|}
\hline & Region & BA & $\mathrm{x}$ & $\mathrm{y}$ & $\mathrm{z}$ & $\mathrm{z}$-score & voxels \\
\hline $\mathrm{L}$ & IFG/Insula & $47 / 13$ & -33 & 20 & -4 & 5.88 & 513 \\
\hline $\mathrm{L}$ & Th/PG & $/ 27$ & -21 & -35 & 2 & 5.51 & \\
\hline $\mathrm{L}$ & Th/Caudate & & -18 & -20 & 15 & 5.37 & \\
\hline $\mathrm{R}$ & $\mathrm{LN}$ & & 21 & -9 & 3 & 5.29 & 266 \\
\hline $\mathrm{R}$ & $\mathrm{LN}$ & & 12 & -3 & -2 & 5.19 & \\
\hline $\mathrm{R}$ & $\mathrm{IFG}$ & 47 & 36 & 20 & -9 & 5.14 & \\
\hline $\mathrm{L}$ & PoG & $2 / 1$ & -53 & -24 & 51 & 5.28 & 537 \\
\hline $\mathrm{L}$ & MFG & 6 & -33 & 2 & 47 & 5.23 & \\
\hline $\mathrm{L}$ & IPL/PoG & $40 / 2$ & -48 & -27 & 43 & 5.12 & \\
\hline $\mathrm{R}$ & Posterior lobe & 18 & 18 & -63 & -32 & 5.24 & 450 \\
\hline $\mathrm{L} / \mathrm{R}$ & Declive & & 0 & -74 & -16 & 5.12 & \\
\hline $\mathrm{R}$ & LG & 18 & 9 & -79 & 1 & 5.11 & \\
\hline $\mathrm{R}$ & Culmen & & 21 & -47 & -15 & 5.06 & 90 \\
\hline $\mathrm{R}$ & Declive/FG/PG & 19 & 27 & -59 & -7 & 4.94 & \\
\hline $\mathrm{R}$ & LG/FG & $18,19 / 19$ & 24 & -70 & -4 & 4.91 & \\
\hline
\end{tabular}

During the recall session, fMRI data were acquired. Subjects solved 40 Boolean problems (eight problems for each rule). Forty tasks were presented to the subject during each fMRI session. Stimuli were presented in a randomized, non-stationary, probabilistic sequence. Elements of each trial were presented in sequential order: red fixation star, conditions items of Boolean task, Boolean task, and white cross (duration of star, conditions, rule, and cross stimuli presentation was $2 \mathrm{~s}, 2 \mathrm{~s}, \mathrm{RT} \leq 6 \mathrm{~s},(8+6-\mathrm{RT}) \mathrm{s}$, respectively) (Fig. 1). The task 
presentation participants were asked to retrieve the correct answer from stable memory, and press " 1 " or " 0 " on two button boxes using the right index and middle fingers. Reaction times were obtained for each trial. Subsequent to pressing the button, the next trial took place following an inter-trial interval. Stable memory tasks were assumed to primarily involve the hit retrieval of mathematic facts and template knowledge stored in long-term memory, without online calculation. Therefore, a white cross was utilized as a baseline condition.

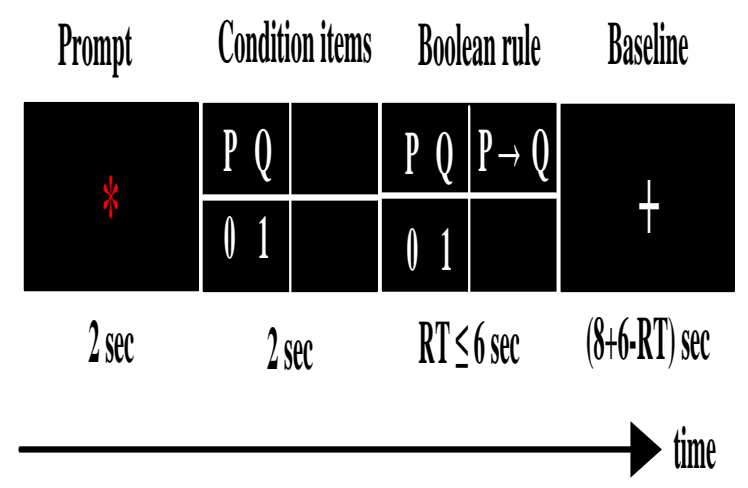

Fig. 1. Example of a trial

\subsection{Image acquisition and analysis}

Data acquisition. Imaging was performed on a 3.0-Tesla Siemens Scanner with a receive-only whole head coil. Prior to anatomic imaging, BOLD data were acquired by an echo-planar imaging (EPI) sequence, and with AC-PC (anterior commissure-posterior commissure) on the twelfth slice from the bottom. Imaging parameters were as follows: slices $=30$, TR/TE $=2000 / 31 \mathrm{~ms}$, slice thickness $=4 \mathrm{~mm}$, slice gap $=0.8 \mathrm{~mm}$, flip angle $=90^{\circ}, \mathrm{FOV}=256 \times 256 \mathrm{~mm}^{2}$, matrix $=64 \times 64,0.8 \times 0.8 \times 4.8 \mathrm{~mm}^{3}$ spatial resolution. For each subject, the fMRI scan during run state lasted for $12 \mathrm{~min} 10 \mathrm{~s}$ and 362 volumes were obtained. In a structural session, whole head T1 weighted images were acquired $\left(\mathrm{TR} / \mathrm{TE} / \mathrm{TI}=130 / 2.89 / 800 \mathrm{~ms}\right.$, slices $=30$, flip angle $=70^{\circ}$, matrix $=320 \times 320$, slice thickness $=4 \mathrm{~mm}$, slice gap $=0.8 \mathrm{~mm}, \mathrm{FOV}=256 \times 256 \mathrm{~mm}^{2}$ ) in the same position as the functional images.

Image preprocessing was conducted using SPM2 package (www.fil.ion.ucl.ac.uk/spm) implemented in Matlab 6.5. The first 2 volumes $(10 \mathrm{~s})$ of each subject were discarded to allow for magnetic saturation effects. The remaining 360 functional scans were first corrected for differences in slice acquisition time and realigned to the first volume to correct for inter-scan movements [13]. Movement parameters during spatial realignment were not greater than $3 \mathrm{~mm}$ and $3^{\circ}$ for the more analysis.

Data analysis. The structural T1-weighted volumes were coregistered to the mean EPI volume and spatially normalized to a standard $\mathrm{T} 1$ template in the space of Talairach. These normalization parameters of normalizing the structural volume were reapplied to the $\mathrm{T} 2 *$-weighted volumes for performing spatial normalization. The functional volumes were spatially normalized to a standard EPI template. During normalization, all volumes were resampled in $3 \times 3 \times 3 \mathrm{~mm}^{3}$ voxels. Finally, all T2*-weighted volumes were smoothed with a 6-mm full width at half-maximum isotropic Gaussian kernel after spatial normalization.

The fMRI data were statistically analyzed using the general linear model (GLM) and statistical parametric mapping. Since the Boolean tasks were trained in a time scheme, including seven different training sessions to consolidate rule memory, practice-related changes collapsed these trials in a range of seven days as the 
stabilized condition. The explanatory variables (stability-based hits, trials of no interest comprising of all trials that took longer than $6 \mathrm{~s}$, and intertrial intervals) were temporally convolved with the canonical hemodynamic response function (HRF) along with its temporal derivatives provided by SPM2. Regressor of the intertrial interval was used as the explicit baseline activation. Temporal filtering was achieved using autoregressive modeling (AR1) to model high-frequency noise, and a high pass filter was implemented using a cut-off period of $128 \mathrm{~s}$ in order to remove the low-frequency effects from the time series. These first-level results from $\operatorname{SPM}\{\mathrm{t}\}$ contrast images were used in second-level random-effects analyses. At the second level, initially a one-sample t-test was calculated with a threshold of $\mathrm{P}<0.05$ (FWE (family-wise error)-corrected), and then the cluster-size statistics were used as the test statistic applying a threshold of $\mathrm{P}<0.00001$ (corrected, minimum cortex volume $\geq 80$ voxels cluster size). This analysis was limited to the brain cortex that was active for the stabilized condition. For the anatomical location of significant clusters, we transformed MNI (Montreal Neurological Institute) template to Talairach space [14]. The Talairach atlas as implemented by the Talairach Daemon [15] was used as reference template for corresponding Brodmann's Areas. Finally, we performed a region of interest (ROI) analysis in the parietal and the frontal associative cortex volume described above, in each of the task conditions compared to the average baseline.

\section{Results}

\subsection{Behavioural data}

Table 2 shows probabilities of correct responses and reaction time (RT) for the hit trials of pre-test (nonmemory), post-test (labile memory) and long-test (stabilized memory) in three fMRI Experiments 1, 2 and 3. In the study phase, accuracy in detecting stability (Boolean rules) was high, with mean rates of $97.78 \%$ in long-test (the stabilized memory test) and $95.97 \%$ in post-term test (the labile memory test). Accuracy was not significant different between the long-test (stabilized) and post-test (labile).

Demands may have been relatively greater in the labile test (post-test), given that there was a greater need to encode the rules. In line with this idea, stability detection was significantly less reaction time in long-test (the stabilized memory test) than in post-test (the labile memory test) $(2.35 \mathrm{~s}$ vs.1.66 s, respectively, $\mathrm{t}=4.22$, $\mathrm{p}<0.001)$.

\section{2. fMRI data}

FMRI was used to investigate memory stability under the condition of known background knowledge. The fMRI trail (e.g. $\mathrm{P} \rightarrow \mathrm{Q}$ ) was simpler than the training trail (e.g. $\mathrm{P} \rightarrow \mathrm{Q} \uparrow \mathrm{P}$ ), which took place outside of the scanner and expected those operations were manipulated. The subject performance task in

L: left; R: right; BA: Brodmann's regions; IFG: Inferior frontal gyrus; Th: Thalamus; PG: Parahippocampal gyrus; LN: Lentiform Nucleus; IFG: Inferior frontal gyrus; PoG: Postcentral gyrus; MFG: Middle frontal gyrus; IPL: Inferior parietal lobule; LG: Lingual gyrus.

the fMRI scanner comprised the stability effects of over- learning responses, which was responsible for the effect activity correlated with the fact memory task rule. The subjects were not required to understand visual objects, but to rather retrieve a rule, which was considered related to maintenance, and not calculation, processes.

Consistent with previous studies [16, 17], memory stability-dependent activity was obtained for single features of rule representation, as well as retrieval in the left inferior parietal lobule and left inferior frontal gyrus. Activity in the inferior parietal lobule (Fig. 2A) was also dependent on stabilized memory for all rules. When the core systems of number were compared with stable memory of Boolean rules, the activation on the same regions was observed within the left inferior frontal gyrus (Fig. 2B). When retrieval rules the activation regions were obtained within whole brain (Fig. 2C). 


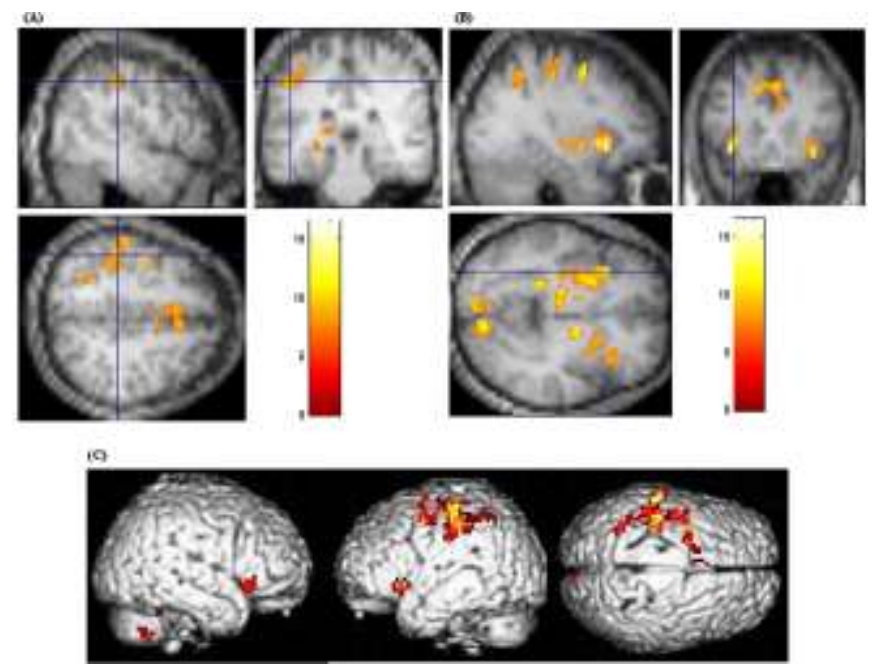

Fig. 2 (A) Activation in the left inferior frontal gyrus (B) activation in the left inferior parietal lobule (C) Three-dimensional rendering of significant cortical activation

\section{Discussion}

The present study provided evidence that experience-dependent stability in human mathematics was accompanied by modulations within the network of regions recruited during encoding. First, abstract mathematical rules, which require stronger retrieval capacity, and stress symbolic and concrete depictions were linked together in the processing regions, which should exhibit larger brain activity in fronto-parietal regions compared with number-processing tasks that merely induce recall of exact arithmetic facts. These regions have been repeatedly observed in response to relational numerical calculation tasks in adults [17,18], and have been shown display differential activation depending on the arithmetic problems [19]. Previous lesion and imaging studies of arithmetic operations have demonstrated the importance of the inferior parietal lobule in arithmetic processing [20]. Exact arithmetical facts (e.g., 2+3) are stored and maintained in same, but have also been associated with inferior frontal [21] and subcortical areas [22]. Second, previous studies have demonstrated the contribution of language in the retrieval of arithmetic facts [23]. Some studies have proposed that the parietal brain circuit is associated with language production and comprehension for storing and retrieving arithmetic facts [3]. Both hypotheses have been verified, including similar modifications of brain activation for all trained rules and activation associated with the core systems of number. When more response regions from these rules were compared with arithmetic facts, results confirmed that complex tasks proceed through more cognitive steps. Boolean rules vs. number-processing tasks previous studies were compared with identify common activations. fMRI data suggested that the partial cortex interacts with other regions to format of stabilized memory, which involved the extending of processing of arithmetic facts [17]. Although former studies did not compare advanced mathematics to basic number-processing, the present results suggested that the fronto-parietal regions may serve to increase knowledge based on arithmetical facts. For example, Fehr et al. [24] suggested that more difficult arithmetic problems require combined arithmetic facts, which activates the parietal regions [10]. Based on these data, Dehaene et al. [6] proposed that the source of mathematical thinking composes of linguistic and visual-spatial neural circuits, both of which are necessary for arithmetic processing. Activation in the left inferior parietal [25] and left parietal regions [26] has been shown to be activated in number-processing studies. 


\section{Concluding Remarks}

In conclusion, adults with stable memory exhibited the development of mathematical fact knowledge, culture background, and conceptual understanding, which were not mutually exclusive. Based on the model of Feigenson et al.'s [4] model, analyses revealed specific associations between rule processing and background knowledge. Based on previous studies focused on the cultural abilities of number-processing, the present results provided a better understanding for the origins of some key domains of the human mathematic culture.

\section{Acknowledgements}

This work was supported by Doctor Start-up Fund of Harbin Normal University (2010), and Philosophy Social Sciences Program of Heilongjiang Province of China (Nos. 10B037). Furthermore, we are especially grateful to Erik, H.Q. Tu, L.L. Ding and one anonymous reviewer, who gave some suggestions that improved the manuscript.

\section{References}

[1] C. Tomasi, "Past performance and future results," Nature vol. 428, pp. 378, 2004.

[2] V. Kostrubiec, J. Tallet, and P.G. Zanone, "How a new behavioral pattern is stabilized with learning determines its persistence and flexibility in memory," Exp. Brain. Res. vol. 170, pp.238-244, 2006.

[3] S. Dehaene, M. Piazza, P. Pinel, and L. Cohen, "Three parietal circuits for number processing," Cogn Neuropsychol, vol. 20, pp. 487-506,2003.

[4] L. Feigenson, S. Dehaene, and E. Spelke, "Core systems of number," Trends Cogn. Sci., vol. 8, pp. $307-$ 314, 2004.

[5] D. Ansari, "Effects of development and enculturation on number representation in the brain," Nat. Rev. Neurosci., vol. 9,pp. 278-291, 2008.

[6] S. Dehaene, E. Spelke, P. Pinel, R. Stanescu, and S. Tsivkin, "Sources of mathematical thinking: behavioral and brain-imaging evidence," Science, vol. 284, pp. 970-974, 1999.

[7] O. Gruber, P. Indefrey, P. Steinmetz, and A. Kleinschmidt, "Dissociating neural correlates of cognitive components in mental calculation," Cereb Cortex, vol. 11, pp. 350-359, 2001.

[8] H. Ebbinghaus, "Memory: A contribution to experimental psychology," New York: Columbia University Press, 1993.

[9] F. I. M. Craik, and E. Tulving, "Depth of processing and the retention of words in episodic memory," J. Exp. Psychol., vol. 104,pp. 268-294 1975.

[10] L. Trick, and Z. W. Pylyshyn, "Why are small and large numbers enumerated differently? A limited capacity preattentive stage in vision," Psychol. Rev., vol. 101, pp. 80-102, 1994.

[11] M. Delazer, and T. Benke, "Arithmetic facts without meaning," Cortex, vol. 33, pp. 697-710, 1997.

[12] M. H. Sohn, M. V. Albert, K. Jung, C. S. Carter, and J. R. Anderson, "Anticipation of conflict monitoring in the anterior cingulate cortex and the prefrontal cortex," PNAS, vol. 104, pp. 10330-10334, 2007.

[13] K. J. Friston, A. P. Holmes, J. B. Poline, P. J. Grasby, S. C. R. Willisams, R. S. J. Frackowiak, and R. Turner, "Analysis of fMRI time-series revisited," NeuroImage, vol. 2, pp. 45-53, 1995.

[14] J. Talairach, and P. Tournoux, "Co-planar stereotaxic atlas of the human brain," Thieme Medical Publishers, New York, 1988.

[15] J. Lancaster, P. Kochunov, M. Woldorff, M. Liotti, L. Parsons, L. Rainey, D. Nickerson, and P. Fox, "Automatic talairach labels for functional activation sites," NeuroImage, vol.11, pp. S483, 2000.

[16] T. C. Rickard, S. G. Romero, G. Basso, C. Wharton, S. Flitman, and J. Grafman, "The calculating brain: 
an fMRI study," Neuropsychologia, vol. 38, pp. 325-335, 2000.

[17] O. Simon, F. Kherif, G. Flandin, J. B. Poline, D. Riviere, J. F. Mangin, D. Le Bihan, and S. Dehaene, "Automatized clustering and functional geometry of human parietofrontal networks for language, space, and number," Neuroimage, vol. 23, pp. 1192-1202, 2004.

[18] K. Kucian, T. Loenneker, T. Dietrich, M. Dosch, E. Martin, and M. von Aster, "Impaired neural networks for approximate calculation in dyscalculic children: a functional MRI study,” Behav. Brain. Funct., vol. 2, pp. 31, 2006.

[19] L. Zago, M. Pesenti, E. Mellet, F. Crivello, B. Mazoyer, and N. Tzourio-Mazoyer, "Neural correlates of simple and complex mental calculation," NeuroImage, vol. 13, pp. 314-327, 2001.

[20] E. Mayer, M. Reicherts, G. Deloche, L. Willadino-Braga, I. Taussik, M. Dordain, M. Van Der Linden, and J-M. Annoni, "Number processing after stroke: Anatomoclinical correlations in oral and written codes," J. Int. Neuropsychol. Soc., vol. 9, pp. 899-912, 2003.

[21] F. Chochon, L. Cohen, P. F. van de Moortele, and S. Dehaene, "Differential contributions of the left and right inferior parietal lobules to number processing," J. Cogn. Neurosc., vol. 11, pp. 617-630, 1999.

[22] V. Prabhakaran, B. Rypma, and J. D. E. Gabrieli, "Neural substrates of mathematical reasoning: a functional magnetic resonance imaging study of neocortical activation during performance of the necessary arithmetic operations test," Neuropsychol, vol. 15, pp. 115-127, 2001.

[23] L. Zamarian, E. Stadelmann, H. C. Nürk, N. Gamboz, J. Marksteiner, and M. Delazer, "Effects of age and mild cognitive impairment on direct and indirect access to arithmetic knowledge," Neuropsychologia, vol. 45, pp. 1511-1521, 2007.

[24] T. Fehr, C. Code, and M. Herrmann, "Common brain regions underlying different arithmetic operations as revealed by conjunct fMRI-BOLD activation,” Brain Res., vol. 1172, pp. 93-102, 2007.

[25] J. Whalen, M. McCloskey, R. P. Lesser, and B. Gordon, "Localizing arithmetic processes in the brain, evidence from a transient deficit during cortical stimulation,” J. Cogn. Neurosci., vol. 9, pp. 409-417, 1997

[26] H. Thogi, K. Saitoh, H. Takahashi, K. Ustugisawa, H. Yonezawa, K. Hatano, and T. Sasaki, "Agraphia and acalculia after a left prefrontal infarction,” J. Neurol Neurosurg Psychiatry, vol. 58, pp. 629-632, 1995. 